\title{
Exotic Spin Order due to Orbital Fluctuations
}

\author{
W. BrzezICKI ${ }^{a}$, J. DzIARMAGA ${ }^{a}$ AND A.M. OleS $\hat{S}^{a, b}$ \\ ${ }^{a}$ Marian Smoluchowski Institute of Physics, Jagiellonian University, W.S. Reymonta 4, PL-30-059 Kraków, Poland \\ ${ }^{b}$ Max-Planck-Institut für Festkörperforschung, Heisenbergstr. 1, D-70569 Stuttgart, Germany \\ We investigate the phase diagrams of the spin-orbital $d^{9}$ Kugel-Khomskii model for increasing system di- \\ mensionality: from the square lattice monolayer, via the bilayer to the cubic lattice. In each case we find strong \\ competition between different types of spin and orbital order, with entangled spin-orbital phases at the crossover \\ from antiferromagnetic to ferromagnetic correlations in the intermediate regime of Hund's exchange. These phases \\ have various types of exotic spin order and are stabilized by effective interactions of longer range which follow from \\ enhanced spin-orbital fluctuations. We find that orbital order is in general more robust while spin order melts first \\ under increasing temperature, as observed in several experiments for spin-orbital systems.
}

DOI: 10.12693 /APhysPolA.126.A-40

PACS: 75.10.Jm, 03.65.Ud, 64.70.Tg, 75.25.Dk

\section{Introduction}

The spin-orbital physics has started over 50 years ago when Kugel and Khomskii introduced the superexchange model for degenerate $e_{\mathrm{g}}$ orbitals in $\mathrm{KCuF}_{3}$ [1], called the Kugel-Khomskii (KK) model. In cases when degenerate orbitals are partly filled both spins and orbitals have to be treated as quantum variables that are $a$ priori strongly coupled to each other [2]. A similar situation occurs in a number of compounds with active orbital degrees of freedom, where strong on-site Coulomb interaction $U$ localizes charge carriers (electrons or holes) and gives rise to spin-orbital superexchange [3-7]. A principal difficulty in such systems follows from enhanced quantum fluctuations $[8,9]$ which may destabilize long-range magnetic order and could lead either to short-range spinorbital correlations or to new quantum phases. Interplay between spin and orbital interactions stabilizes various types of magnetic order which coexist with particular orbital order, as both in colossal magnetoresistance manganites [10], or in the vanadium perovskites [11]. The theoretical approaches are rare and notoriously difficult [12] - while in one-dimensional (1D) systems an electron can break into a spinon and an orbiton [13, 14], explicit treatment of entangled spin-orbital states is required both in some model systems [15-21], and in realistic models, for instance to describe the physical properties of the vanadium perovskites [22-25]. Therefore, the phase diagrams of such model systems are very challenging and are the subject of active research.

Another route which makes the studies of spin-orbital physics of high interest is frustrated magnetism. Frustrated spin models are known to exhibit very interesting properties and have frequently exotic ground states [26]. In systems with active orbital degrees of freedom such states may arise from intrinsic frustration of orbital interactions which, unlike the spin ones with $\mathrm{SU}(2)$ symmetry, are directional both in the $e_{\mathrm{g}}$ orbital models [27-29] and in the compass model [30-34] - they contain terms which compete with one another. Studies of such models require more sophisticated approaches than the single-site mean field (MF) approximation or linear spin-wave expansion. Exact solutions are possible only for some 1D spin-orbital models [35-38] - they also highlight the importance of quantum effects beyond simple classical approaches.

Coming back to the KK model, it explains the origin of the orbital order in $\mathrm{KCuF}_{3}$ which is responsible for the onset of the $A$-type antiferromagnetic $(A-\mathrm{AF})$ order at low temperature [5], and the quasi-1D AF Heisenberg structure with spinon excitations [39-41] at high temperature. In spite of strong interplay between the spin and orbital degrees of freedom, the energy scales separate and the orbital order occurs in $\mathrm{KCuF}_{3}$ below the structural phase transition at rather high temperature, $T_{\mathrm{OO}} \approx 800 \mathrm{~K}$. This demonstrates a strong Jahn-Teller coupling between the orbitals and lattice distortions [27], which plays also an important role in $\mathrm{LaMnO}_{3}$ [42] and cannot be ignored when the data for real compounds are explained. It has been also realized that the so-called Goodenough processes [5, 43, 44], involving excitations on oxygen (ligand) sites, do contribute to the superexchange in charge-transfer insulators, and the structure of the effective Hamiltonian is richer than that of the KK model. However, we shall consider here just the spinorbital superexchange models as they arise in the original derivation [45] from the multiband Hubbard model [46].

While the coexisting $A$-AF order and the $C$-type orbital order $\left(C\right.$-OO) are well established in $\mathrm{KCuF}_{3}$ below the Néel temperature $T_{\mathrm{N}} \approx 39 \mathrm{~K}$ and this phase is reproduced by the spin-orbital $d^{9}$ superexchange model in the MF approximation [45], the phase diagram of this model is still unknown beyond the MF approach because of strongly coupled spin and orbital degrees of freedom, and poses an outstanding question in the theory: Which types of coexisting spin and orbital order (or disorder) are possible here when the microscopic parameters: (i) crystal-field (CF) splitting of the $e_{\mathrm{g}}$ orbitals $E_{z}$, and (ii) Hund's exchange $J_{\mathrm{H}}$, are varied? It has been suggested that the long-range AF order is destroyed by spin-orbital 
quantum fluctuations [8], but this is still controversial and also other ordered states stabilized by the order-outof-disorder mechanism might arise [9].

The purpose of this paper is to investigate the phase diagrams of the KK model at increasing dimensionality. Thereby we summarize the results of the earlier studies for a two-dimensional (2D) monolayer [47], bilayer [48], and a three-dimensional (3D) perovskite (cubic) system [49]. We show below that spin-orbital fluctuations and entanglement $[7,15]$ play a very important role here and stabilize exotic types of magnetic order in all these systems. We start with introducing the KK model in Sect. 2. Next in Sect. 3 we explain two standard methods used to investigate the phase stability in different parameter regimes: (i) a one-site MF approximation (Sect. 3.2), and (ii) a cluster MF approximation (Sect. 3.3). We show the essential differences between the phase diagrams obtained in these two methods and argue that new types of exotic spin order arise from the entangled spin-orbital interactions. We also address the types of order found at finite temperature in the $2 \mathrm{D}$ monolayer, where we show that the magnetic order is more fragile even in the absence of the Jahn-Teller coupling. In Sect. 4 we explain the origin of the spin order found in the cluster MF approximation. The paper is summarized in Sect. 5.

\section{Kugel-Khomskii model}

The spin-orbital superexchange KK model was originally introduced for $\mathrm{Cu}^{2+}\left(d^{9}\right)$ ions in the perovskite structure of $\mathrm{KCuF}_{3}$, with $S=1 / 2$ spins and $e_{\mathrm{g}}$ orbital $\tau=1 / 2$ pseudospins [1]. The correct multiplet structure was included only later [45] when it was derived from the degenerate Hubbard Hamiltonian with hopping $t$, intraorbital Coulomb interaction $U$ and Hund exchange $J_{\mathrm{H}}$ for $e_{\mathrm{g}}$ electrons [46]. It describes the Heisenberg SU(2) spin interactions coupled to the orbital problem, with the superexchange constant $J=4 t^{2} / U$,

$$
\begin{aligned}
\mathcal{H} & =-\frac{1}{2} J \sum_{\langle i j\rangle \| \gamma}\left[\left(r_{1} \pi_{\langle i j\rangle}^{t}+r_{2} \pi_{\langle i j\rangle}^{s}\right)\left(\frac{1}{4}-\tau_{i}^{\gamma} \tau_{j}^{\gamma}\right)\right. \\
& \left.+\left(r_{2}+r_{4}\right) \pi_{\langle i j\rangle}^{s}\left(\frac{1}{2}-\tau_{i}^{\gamma}\right)\left(\frac{1}{2}-\tau_{j}^{\gamma}\right)\right]+\mathcal{H}_{0},
\end{aligned}
$$

where the CF splitting term,

$$
\mathcal{H}_{0}=E_{z} \sum_{i} \tau_{i}^{z},
$$

lifts the orbital degeneracy, and the coefficients,

$$
\begin{gathered}
r_{1}=\frac{1}{1-3 \eta}, \quad r_{2}=\frac{1}{1-\eta}, \quad r_{4}=\frac{1}{1+\eta}, \\
\left(r_{3}=r_{2}\right) \text { depend on Hund exchange }[45], \\
\eta \equiv \frac{J_{\mathrm{H}}}{U} .
\end{gathered}
$$

Here $\gamma=a, b, c$ is the bond direction along one of the cubic axes $\gamma$. In a bilayer two $a b$ planes are connected by interlayer bonds along the $c$ axis [48], while a monolayer has only bonds within a single $a b$ plane [47], i.e., $\gamma=a, b$. In all these cases spin interactions are described with the help of spin projection operators on a triplet or a singlet configuration on a bond $\langle i j\rangle$,

$$
\begin{aligned}
& \pi_{\langle i j\rangle}^{s}=\frac{1}{4}-\boldsymbol{S}_{i} \cdot \boldsymbol{S}_{i+\gamma}, \\
& \pi_{\langle i j\rangle}^{t}=\frac{3}{4}+\boldsymbol{S}_{i} \cdot \boldsymbol{S}_{i+\gamma},
\end{aligned}
$$

and $\tau_{i}^{\gamma}$ are the orbital operators for the bond direction $\gamma=a, b, c$. They are defined in terms of Pauli matrices in the orbital space, $\left\{\sigma_{i}^{x}, \sigma_{i}^{z}\right\}$, as follows:

$$
\begin{aligned}
& \tau_{i}^{a(b)} \equiv \frac{1}{4}\left(-\sigma_{i}^{z} \pm \sqrt{3} \sigma_{i}^{x}\right), \\
& \tau_{i}^{c} \equiv \frac{1}{2} \sigma_{i}^{z}=\frac{1}{2}\left(n_{i z}-n_{i x}\right) .
\end{aligned}
$$

These operators act in the orbital space, with the basis states

$$
|z\rangle \equiv\left(3 z^{2}-r^{2}\right) / \sqrt{6}, \quad|x\rangle \equiv\left(x^{2}-y^{2}\right) / \sqrt{2},
$$

and the $|z\rangle(|x\rangle)$ orbital is an "up" ("down") orbital state. Finally, $E_{z}$ in Eq. (2) is the crystal-field (CF) splitting of $e_{\mathrm{g}}$ orbitals which favors either $|z\rangle$ (if $E_{z}<0$ ) or $|x\rangle$ (if $E_{z}>0$ ) orbital state occupied by a hole at each site $i$. Thus the model Eq. (1) depends on two parameters: $E_{z} / J$ and Hund exchange $\eta(4)$ - we vary them below to determine the phase diagrams analyzed in Sect. 3 .

\section{Mean-field phase diagrams}

\subsection{Spin and orbital order}

We consider spin and orbital order with up to two sublattices, as well as phases composed of equivalent cubes (or plaquettes in the 2D system). The spin order is simpler due to the $\mathrm{SU}(2)$ symmetry of the Heisenberg interactions, as it gives $\left\langle S_{j}^{z}\right\rangle= \pm 1 / 2$ when the $z$-th spin components are chosen to determine the broken symmetry state, so it suffices to consider AF or FM bonds in various nonequivalent phases with long-range spin order. The spin $\mathrm{SU}(2)$ interaction is replaced in the MF approximation by local operators at site $i$ interacting with the MF values for the operators at neighboring sites $j$,

$$
\boldsymbol{S}_{i} \cdot \boldsymbol{S}_{j} \simeq S_{i}^{z}\left\langle S_{j}^{z}\right\rangle+\left\langle S_{i}^{z}\right\rangle S_{j}^{z}-\left\langle S_{i}^{z}\right\rangle\left\langle S_{j}^{z}\right\rangle
$$

We consider the magnetic phases depicted in a schematic way in Fig. 1, where $\left\langle S_{j}^{z}\right\rangle \neq 0$ : (i) $A$-AF phase — with FM order in the $a b$ planes and AF correlations along the $c$ axis (Fig. 1i), (ii) $C$-AF phase - with AF order in the $a b$ planes and FM correlations along the $c$ axis (Fig. 1ii), (iii) FM phase (Fig. 1iii), and (iv) G-AF phase Néel state (Fig. 1iv). The first two phases, (i) $A-\mathrm{AF}$ and (ii) $C$-AF phase, contain partly FM bonds which are stabilized by the orbital order (OO).

On the contrary, due to the absence of SU(2) symmetry, the $\mathrm{OO}$ in the present $\mathrm{KK}$ models may involve not only one of the two basis $e_{\mathrm{g}}$ orbital states $\left.\{|z\rangle, x\rangle\right\}$, but also their linear combinations,

$$
|\theta\rangle=\cos \left(\frac{\theta}{2}\right)|z\rangle+\sin \left(\frac{\theta}{2}\right)|x\rangle,
$$

parameterized by an angle $\theta$ which defines their amplitudes at site $i$. In the orbital sector we apply then the MF 
decoupling for the products $\left\{\tau_{i}^{\gamma} \tau_{i \pm \gamma}^{\gamma}\right\}$ along the axis $\gamma$ :

$$
\tau_{i}^{\gamma} \tau_{i \pm \gamma}^{\gamma} \simeq\left\langle\tau_{i}^{\gamma}\right\rangle \tau_{i \pm \gamma}^{\gamma}+\tau_{i}^{\gamma}\left\langle\tau_{i \pm \gamma}^{\gamma}\right\rangle-\left\langle\tau_{i}^{\gamma}\right\rangle\left\langle\tau_{i \pm \gamma}^{\gamma}\right\rangle \text {. }
$$

As order parameters we take $t^{a} \equiv\left\langle\tau_{1}^{a}\right\rangle$ and $t^{c} \equiv\left\langle\tau_{1}^{c}\right\rangle$ for a chosen site $i=1$ (which is sufficient in orbital sector as $t^{b}=-t^{a}-t^{c}$ ) and we assume two orbital sublattices: each neighbor of the site $i$ is rotated by $\pi / 2$ in the $a b$ plane meaning that $\left\langle\tau_{i+\gamma}^{a(b)}\right\rangle=t^{b(a)}$. A frequently encountered form of the $\mathrm{OO}$ is a two-sublattice structure, with two orbitals given by angles $\theta_{A}=\theta$ and $\theta_{B}=-\theta$ :

$$
\begin{aligned}
\left|\theta_{A}\right\rangle_{i} & =\cos \left(\frac{\theta}{2}\right)|z\rangle_{i}+\sin \left(\frac{\theta}{2}\right)|x\rangle_{i}, \\
\left|\theta_{B}\right\rangle_{j} & =\cos \left(\frac{\theta}{2}\right)|z\rangle_{j}-\sin \left(\frac{\theta}{2}\right)|x\rangle_{j} .
\end{aligned}
$$

Examples of simple $\mathrm{OO}$ which may a priori coexist with spin order are shown as well in Fig. 1: alternating orbital (AO) order with either $x$-like or $z$-like orbitals in (a) and (b), and two ferro-orbital (FO) orders with either $z$ or $x$ orbitals occupied at each site, in (c) and (d). In reality the angles for the AO states vary in a continuous way as functions of the CF parameter $E_{z} / J$, and therefore an efficient way of solving the one-site MF equations consists of assuming possible magnetic orders and for each of them deriving the effective orbital-only model. Such models are next compared and the phases with the lowest energy are found, as presented below for the case of the 2D monolayer, [47], the KK bilayer [48], and for the $3 \mathrm{D}$ cubic model [49].
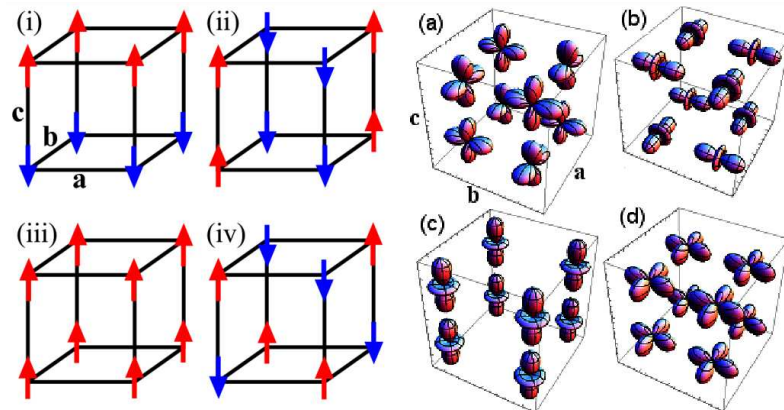

Fig. 1. Possible types of one- and two-sublattice spin and orbital order in a cubic system. Left part schematic view of four representative spin phases (arrows stand for up or down spins): (i) $A-\mathrm{AF}$, (ii) $C-\mathrm{AF}$, (iii) FM, and (iv) $G$-AF one. Right part - schematic view of four types of orbital order on a cube of the 3D (bilayer) lattice: (a) AO order with $\left\langle\tau_{i}^{a(b)}\right\rangle=1 / 2$ changing from site to site and $\left\langle\tau_{i}^{c}\right\rangle=-1 / 4$, obtained for $E_{z}<0$, (b) AO order with $\left\langle\tau_{i}^{a(b)}\right\rangle=-1 / 2$ changing from site to site and $\left\langle\tau_{i}^{c}\right\rangle=1 / 4$, obtained for $E_{z}>0$, (c) FO order with occupied $z$ orbitals and $\left\langle\tau_{i}^{c}\right\rangle=1 / 2$, and (d) FO order with occupied $x$ orbitals and $\left\langle\tau_{i}^{c}\right\rangle=-1 / 2$.

The simplest approximation to obtain the possible types of order in a spin-orbital model, like the KK models Eq. (1) considered here, is the single-site MF approach which consists of two steps: (i) decoupling of spin and orbital interactions, and (ii) subsequent factorization of interactions of both types on the bonds into (spin or orbital) operators at a given site $i$ coupled to the MF terms on its neighboring sites. The values of the projection operators (5) and (6) depend on the assumed spin order, and they may be easily eliminated in this approach when the spin scalar products are replaced by their values in the MF states, being either $\left\langle\boldsymbol{S}_{i} \cdot \boldsymbol{S}_{i+\gamma}\right\rangle_{\mathrm{FM}}=1 / 4$, or $\left\langle\boldsymbol{S}_{i} \cdot \boldsymbol{S}_{i+\gamma}\right\rangle_{\mathrm{AF}}=-1 / 4$. Taking different types of spin order (i)-(iv), and assuming the classical average values of the spin projection operators, one finds the MF equations which are next solved for each of the considered three systems: the $2 \mathrm{D}$ monolayer, the bilayer, and the 3D perovskite. Solutions of the self-consistency equations and ground state energies in different phases can be obtained analytically, as explained on the example of the bilayer system in [48].

\subsection{Single-site mean-field approximation}

The simplest approach is a single-site MF approximation applied to the KK models Eq. (1). It excludes any spin fluctuations as the spin projectors $\pi_{\langle i j\rangle}^{t(s)}\left(\pi_{\langle i j\rangle}^{s}\right)$ are here replaced by their mean values, where the dependence on the bond $\langle i j\rangle$ reduces to direction $\gamma$ in phases with translationally invariant magnetic order shown in Fig. 1. The phase diagrams of the KK model follow from the two competing trends when the parameters, the CF splitting $E_{z} / J$, and Hund exchange $\eta(4)$ are varied. While increasing $\mathrm{CF}$ parameter $E_{z}$ causes a switch from $z$ to $x$ orbitals, increasing Hund's exchange favors FM interactions along at least the $c$ axis, and coexisting with the AO phase.

Using the factorized form of the spin-orbital superexchange, and the order parameters,

$$
s_{i}^{z} \equiv\left\langle S_{i}^{z}\right\rangle, \quad t_{i}^{\gamma} \equiv\left\langle\tau_{i}^{\gamma}\right\rangle,
$$

with $\left|\left\langle S_{j}^{z}\right\rangle\right|=\frac{1}{2}$, we determined MF energies of all possible phases with spin long-range order and optimized values of the orbital order parameters $\left\{t_{i}^{\gamma}\right\}$. Consider first the case of vanishing Hund exchange $\eta=0$. In this case the multiplet structure (3) collapses to a single level and $r_{i}=1 \forall i$. Therefore the excited states with double occupancies in two different orbitals, e.g. $x_{i}^{1} z_{i}^{1}$ at site $i$, do not introduce any spin dependence of the superexchange as the contributions from the triplet and singlet excitation compensate each other, and the only magnetic term stems from double occupancies at the same $e_{\mathrm{g}}$ orbital. Such terms are AF and resemble the ones derived from the Hubbard model without orbital degeneracy in the $t^{-}$ $J$ model [50]. Therefore, one finds that at $\eta=0$ two AF Néel phases are degenerate: $G-\mathrm{AF} z$ and $G-\mathrm{AF} x$. Actually, individual contributions to the ground state energy from the bonds along the $a b$ axes and along the $c$ axis are quite different in both phases: while the $G$-AF $x$ phase is $2 \mathrm{D}$, with no coupling between the $a b$ planes and is realized for instance in $\mathrm{La}_{2} \mathrm{CuO}_{4}$ [7], the AF superexchange along the $c$ axis is stronger by a factor of 16 than the one along the bonds in the $a b$ planes in the $G-\mathrm{AF} z$ phase. Nevertheless, these energy contributions add to the same 


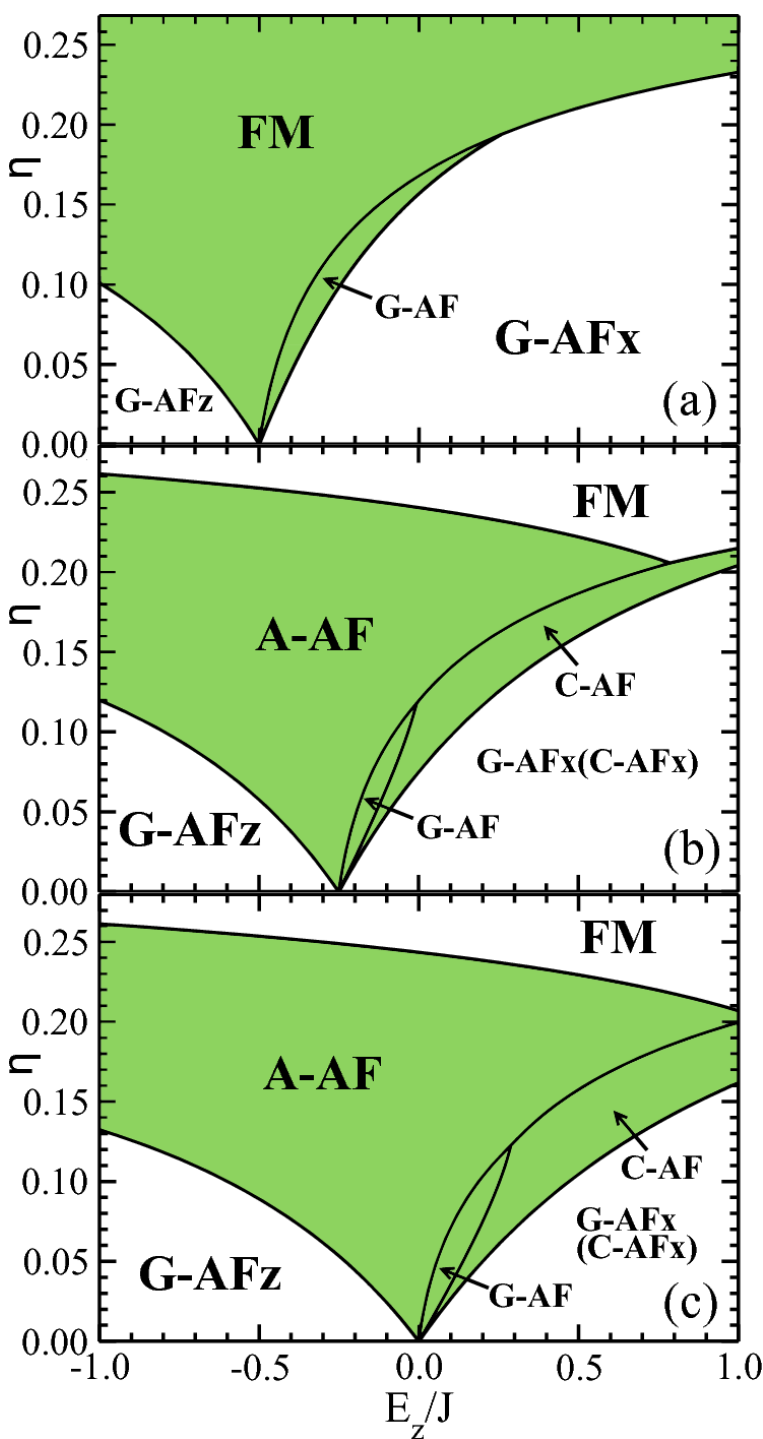

Fig. 2. Phase diagrams of the KK models in the singlesite MF approximation as obtained for: (a) the 2D monolayer [47], (b) the bilayer [48], and (c) the 3D perovskite system [49]. Shaded dark gray (green) areas indicate phases with $\mathrm{AO}$ order: $\mathrm{FM}$ and $G$-AF phase for the monolayer (a), and the $A$-AF, $C$-AF and $G$-AF phases for the bilayer (b) and the 3D perovskite (c) [here the FM phases which coexist also with the AO order are not shadded for clarity]. The remaining $G-\mathrm{AF}$ phases $(G-\mathrm{AF} x$ and $G-\mathrm{AF} z)$ are accompanied by $\mathrm{FO}$ order with fully polarized orbitals, either $x$ (for $E_{z}>E_{z}^{0}$ ) or $z$ (for $\left.E_{z}<E_{z}^{0}\right)$. The quantum critical point with degenerate $G$-AF $x, G$-AF, $A-\mathrm{AF}$, and $G-\mathrm{AF} z$ phases is found at $\left(E_{z}, \eta\right)=\left(E_{z}^{0}, 0\right)$, with decreasing $E_{z}^{0}$ from the 3D perovskite via the bilayer to the $2 \mathrm{D}$ monolayer.

value in a 3D cubic system [45] and the actual occupied orbital (FO order) is decided by the value of the CF splitting. One finds the $G-\mathrm{AF} z$ phase for $E_{z}<0$ and $G-\mathrm{AF} x$ phase for $E_{z}>0$ (Fig. 2c) - they are degenerate at the quantum critical point $\left[\mathrm{QCP}\left(E_{z}^{0}, \eta\right)=(0,0)\right]$. This reflects the cubic symmetry of the model (1) at $E_{z}=0$.
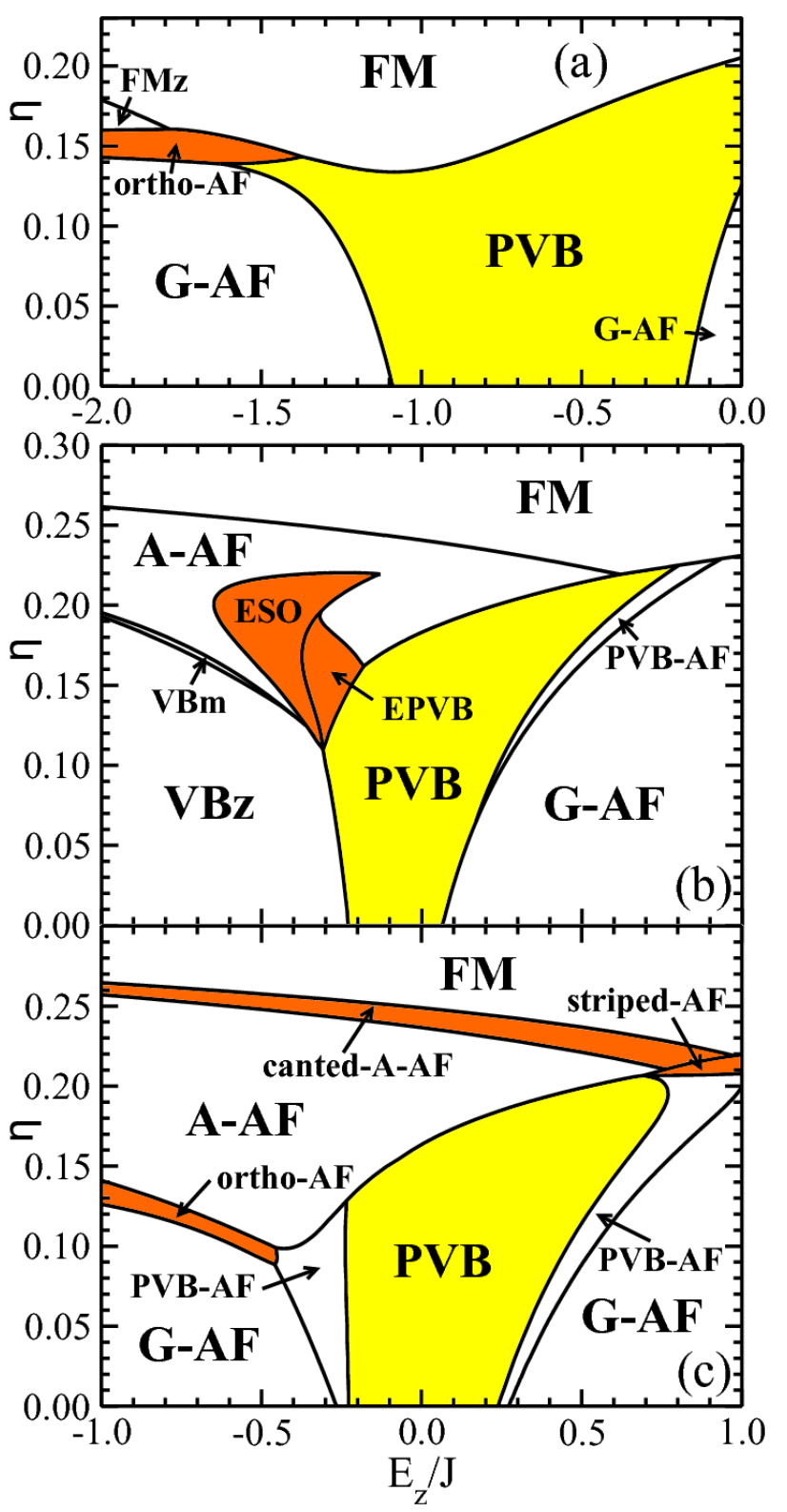

Fig. 3. Phase diagrams of the KK models in the cluster MF approximation as obtained for: (a) the 2D monolayer [47], (b) the bilayer [48], and (c) the 3D perovskite system [49]. Plaquette valence-bond (PVB) phase with alternating spin singlets in the $a b$ planes is highlighted in light gray (yellow) - it occurs between the phases with long-range magnetic order, and the QCP is hidden. When strong competition between AF and FM interactions occurs at increasing $\eta$, new phases with exotic spin order are found - they are highlighted in dark gray (orange).

In two other cases, the cubic symmetry is broken and the transition between the $G-\mathrm{AF} z$ and $G-\mathrm{AF} x$ phase (the QCP) occurs now at finite $E_{z}: E_{z}^{0}=-0.25 J$ and $E_{z}^{0}=-0.5 J$ for the bilayer (Fig. 2b) and for the 2D monolayer (Fig. 2a). This follows from the anisotropic superexchange in the $G$-AF phases - the magnetic MF 
energy decreases rapidly in the $G$-AF $z$ phase with weak exchange bonds and has to be compensated by the $\mathrm{CF}$ term.

Having two degenerate phases at $\left(E_{z}^{0}, 0\right)$ in any of the considered cases, it suffices to add an infinitesimal Hund exchange $\eta>0$ to destabilize the Néel AF order in favor of the $A$-AF phase, with FM interactions in the $a b$ planes and $\mathrm{AF}$ ones along the $c$ axis. Such anisotropic magnetic interactions are supported by the $\mathrm{AO}$ order, with two different orbitals along each bond $(i j) \in a b$, as shown in Fig. 1a. The occupied orbitals belong to two sublattices $A$ and $B$, as given in Eq. (13). One may wonder whether the $G$-AO order depicted in Fig. 1a coexists indeed with the $A$-AF order, and the answer to this question goes beyond the superexchange model (1). In fact, a small interaction with the lattice (ignored in the present analysis) selects one of these phases as the orbital alternation implies lattice distortions around each $\mathrm{Cu}^{2+}$ ion. Such distortions occur within the $a b$ planes and a better energy is found when the planes are repeated along the $c$ axis - this implies the $C$-AO order coexisting with the $A$-AF phase.

As expected, the spin order is $G$-AF when the CF term is large and favors one of the two orbitals, while FM interactions appear when the OO changes to AOs and the spin interactions in the $a b$ planes become FM. In contrast to the FO phases, the $\mathrm{AO}$ order in the shaded phases in Fig. 2 is more involved and the angle $\theta$ in Eq. (13) is selected by the energy minimization in the respective phase. In most cases the transitions between the $G$-AF and $A-\mathrm{AF}$ (or $C-\mathrm{AF}$ or $\mathrm{FM}$ ) are first order. The $A$ AF phase, stable here in a broad range of parameters both in the bilayer (Fig. 2b) and in the 3D perovskite (Fig. 2c), develops from the FM phase in the case of the $2 \mathrm{D}$ monolayer (Fig. 2a). In the two former systems another transition to the (anisotropic) FM phase occurs at large $\eta \approx 0.25$, when the AF terms in the superexchange terms are dominated by triplet charge excitations. In all the cases the $G$-AF spin order occurs as a precursor of the $A$-AF (FM) phase, when $E_{z}$ is decreased and the $a b$ planes become weakly coupled by the $\mathrm{AO}$ order. The $C$-AF phase occurs in addition in a narrow range of parameters in between the $G$-AF $x$ and the $A$-AF phase in Fig. $2 \mathrm{~b}$ and c. This phase is unexpected and suggests that the phase diagrams derived in better approximations might be quite different.

\subsection{Cluster mean-field approximation}

Knowing that spin-orbital quantum fluctuations are enhanced near orbital degeneracy [8], it is of crucial importance to include them when the phase diagrams of the KK models are investigated. Exact diagonalization gives exact ground states of finite clusters and may be combined with MF approach when a cluster under consideration is in contact with its neighboring clusters via the MF terms. We developed this so-called cluster MF approximation for the KK models by embedding a foursite cluster in the plaquette MF (PMF) used in the $2 \mathrm{D}$ model [47], and a cube in the bilayer case [48]. The most natural choice for the $3 \mathrm{D}$ perovskite is a cube as well, but here we limit ourselves to four-site clusters [49] (a plaquette or a linear cluster) to avoid tedious numerical analysis.

The interactions along bonds which belong to a cluster considered in each case are treated by exact diagonalization, while the bonds which couple the cluster with its neighbors are decoupled in the MF approximation. In this way we arrive at the self-consistent MF equations for the order parameters

$$
s_{i}^{\alpha} \equiv\left\langle S_{i}^{\alpha}\right\rangle, \quad t_{i}^{\gamma} \equiv\left\langle\tau_{i}^{\gamma}\right\rangle, \quad v_{i}^{\alpha, \gamma} \equiv\left\langle S_{i}^{\alpha} \tau_{i}^{\gamma}\right\rangle .
$$

Here we consider two spin components, $\alpha=x, z$, as the $\mathrm{SU}(2)$ symmetry of the spin interactions may be broken in a more general way to include some exotic types of magnetic order obtained in particular for the $2 \mathrm{D}$ monolayer [47]. The mixed order parameters $\left\{v_{i}^{\alpha, \gamma}\right\}$ are essential here and influence the stability of phases by including on-site spin-orbital entanglement [48].

The essential qualitative difference between the cluster MF (Fig. 3) and the single-site MF approach (Fig. 2) is the possibility of spin disorder, realized in between the phases with spontaneously broken symmetry. De facto, the phases with long-range spin order $(G-\mathrm{AF}$ and $A-\mathrm{AF}$ ones) which coexist at the QCPs are replaced by the plaquette valence-bond (PVB) states, as shown in Fig. 3. These states are characterized by local order on individual plaquettes within the $a b$ planes, with spin singlets coexisting with pairs of directional orbitals, $3 x^{2}-r^{2}$ or $3 y^{2}-r^{2}$, along the same bonds, and rather weak coupling between them. The orbital states behave more classically and fluctuations between different orientations of singlets are blocked on each plaquette by the MF terms which couple this plaquette to its neighbors. By considering different possible covering of the lattice by such PVB states we could establish their alternation within the $a b$ planes, with each pair of neighboring plaquettes forming a superlattice of plaquettes with alternating horizontal and vertical singlet bonds. These states are particularly robust in the $2 \mathrm{D}$ monolayer and suppress the FM order in a broad regime of parameters, as they are not disturbed here by the perpendicular bonds along the $c$ axis, present in the bilayer and in the 3D perovskite.

The QCPs in the single-site MF approximation and the superlattice of alternating plaquettes realized in the cluster MF may be seen as indications of frustrated spinorbital interactions. It is surprising that this frustration leads not only to spin disorder but also to rather exotic types of spin order, in particular in the $2 \mathrm{D}$ monolayer and in the $3 \mathrm{D}$ perovskite lattice. In both cases the ortho- $\mathrm{AF}$ phase is found in between the $G$-AF and $A-\mathrm{AF}$ phases, and in the $3 \mathrm{D}$ model in addition also the striped-AF and canted- $A$-AF phases are stable in the vicinity of the FM order. Although the present KK models contain only nearest-neighbor (NN) superexchange interactions, when the AF and FM contributions compete, the usual NN superexchange becomes ineffective and other higher order processes contribute [47, 49], as explained below in 
Sect. 4. The case of the bilayer is different as the bonds along the $c$ axis contribute here with the full spin singlet energy when the CF term $\left(E_{z}<0\right)$ selects the $z$ orbitals. This dominates over the other terms along the $a b$ bonds and favors phases with spin disorder in this regime of parameters: entangled spin-orbital (ESO) phase and entangled PVB (EPVB) phase [48], shown in Fig. 3b.

\subsection{Phase diagrams for the monolayer at $T>0$}

Before analyzing spin-orbital entangled states at temperature $T=0$, we emphasize that the spin and orbital interactions concern quite different energy scales. As an illustrative example we consider the $2 \mathrm{D}$ monolayer, where the phase diagrams obtained at finite $T$ demonstrate that the spin order is robust only in two cases: (i) the $G-\mathrm{AF}$ order due to large superexchange at $E_{z}>0$, or (ii) the FM order stabilized by large Hund's exchange $\eta>0.2$. The other spin ordered phases are influenced stronger by thermodynamic spin fluctuations because the exchange interactions are much weaker in them. The exotic orthoAF phase disappears already at $T=0.05 J$ - the paramagnetic (PM) spins are then accompanied by the FO order of the $z$ states, selected by the CF term $E_{z}<0$, see Fig. 4a. In contrast, both phases with spin long-range $G$-AF and $\mathrm{FM} z$ order which are away from the $\mathrm{AF} \leftrightarrow \mathrm{FM}$ transition in the superexchange, are more stable.

Due to the orbital shape, the exchange interactions between pairs of $z$ orbitals are much weaker, and both phases with this type of FO order, the $G-\mathrm{AF} z$ and $\mathrm{FM} z$ phase, are not found at $T=0.20 J$, see Fig. 4b. Here a new PM phase with AO order (PM/AO phase) appears in cases when Hund's exchange is not strong enough to give robust FM order. At the same time the PVB phase shrinks as the spins are too weakly coupled to form stable spin singlets. Even larger regimes of the PM and PM/AO phase are found when temperature is further increased to $T=0.34 J$, see Fig. 4c.

\section{Spin-orbital entangled states \\ 4.1. Origin of exotic magnetic orders}

We explain the origin of the exotic magnetic order on the example of the ortho-AF phase in the $2 \mathrm{D}$ monolayer. This phase occurs in between the $G-\mathrm{AF} z$ phase and the FM phase (either $\mathrm{FM} z$ or with the AO order), see Fig. 3a. Large negative $C F$ splitting, $E_{z}<0$, favors there $z$ orbitals, and the CF term (Eq. (2) may be treated as the unperturbed part of the Hamiltonian), while the superexchange $\propto J$ is the perturbation, $\mathcal{V} \equiv \mathcal{H}-\mathcal{H}_{0}$. The ground state $|0\rangle$ of $\mathcal{H}_{0}$ is the $\mathrm{FO} z$ state with $z$ orbitals occupied by a hole at each site, $\tau_{i}^{c}|0\rangle=\frac{1}{2}|0\rangle$, and the spin order is undetermined. Orbital excitations have a large gap and the ratio $J /\left|E_{z}\right| \equiv\left|\varepsilon_{z}\right|^{-1}$ is a small parameter which may be used here to construct the expansion in powers of $\left|\varepsilon_{z}\right|^{-1}$,

$$
H_{s} \simeq J\left\{H_{s}^{(1)}+H_{s}^{(2)}+H_{s}^{(3)}\right\} .
$$

The terms $H_{s}^{(n)}$ are spin interaction in the $n$-th order of this perturbative expansion. The first order term is an

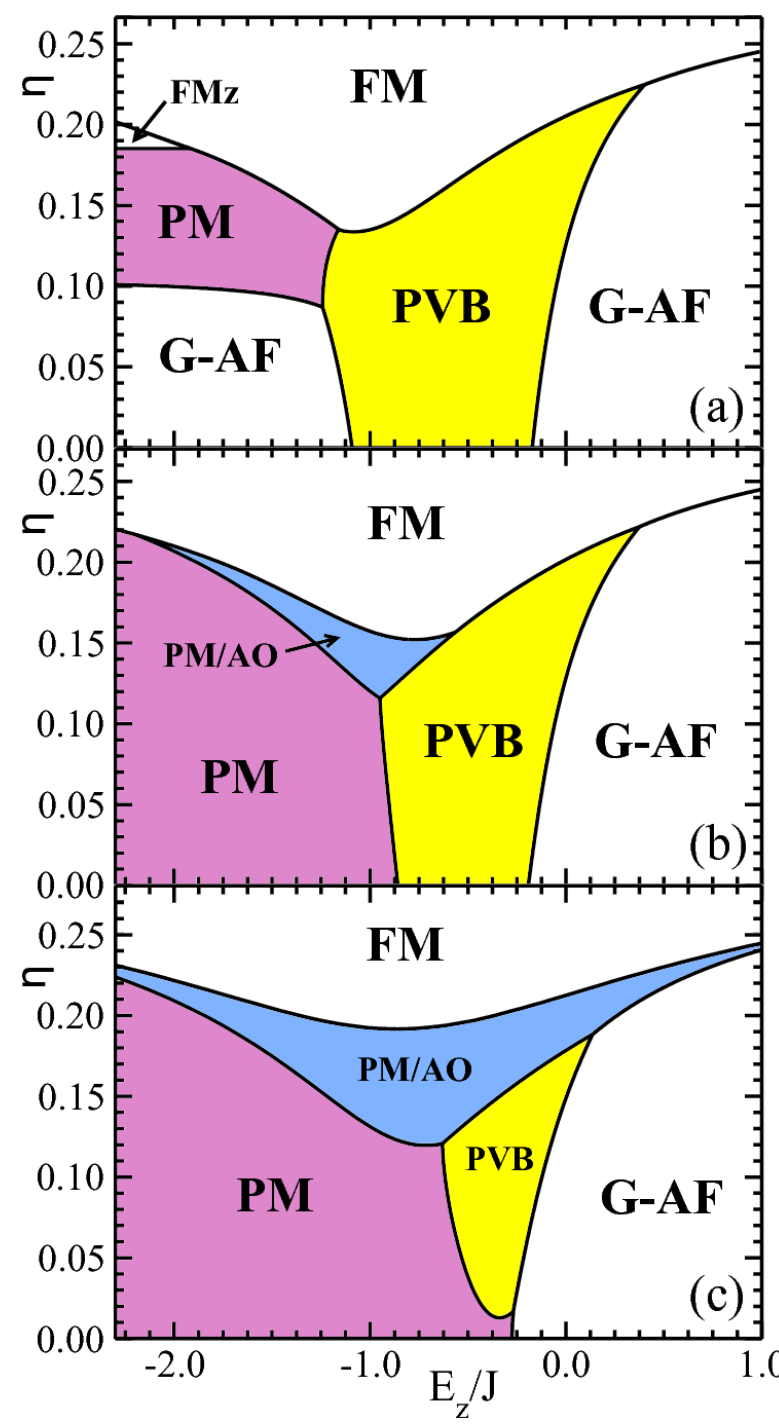

Fig. 4. Evolution of the phase diagram of the 2D KK model with increasing temperature: (a) $T=0.05 \mathrm{~J}$, (b) $T=0.20 J$, and (c) $T=0.34 J$. The spin order changes gradually to paramagnetic (PM) under increasing temperature while the $\mathrm{OO}$ is more robust.

average $H_{s}^{(1)} \equiv\langle 0|\mathcal{V}| 0\rangle$ which is just the superexchange in Eq. (1), projected on the ground state $|0\rangle$, i.e., obtained for the $\mathrm{FO} z$ state. As expected, this term is the Heisenberg interaction along the bonds $\langle i j\rangle \in a b$,

$$
H_{s}^{(1)}=\frac{1}{2^{5}}\left(-3 r_{1}+4 r_{2}+r_{4}\right) \sum_{\langle i j\rangle}\left(\boldsymbol{S}_{i} \cdot \boldsymbol{S}_{j}\right) .
$$

For $\eta<0.155(\eta>0.155)$ the spin interaction is AF (FM), and as long as no further contributions are evaluated the $\mathrm{AF} \leftrightarrow \mathrm{FM}$ transition takes place at $\eta_{0}=0.155$.

Yet, the magnetic order is determined by higher order $H_{s}^{(2)}$ and $H_{s}^{(3)}$ terms for $\eta_{0} \approx 0.155$. These interactions involve more than two sites and are obtained by considering all excited states $|n\rangle$, with orbitals flipped from $z$ to $x$ - they can be evaluated from the matrix elements involving excited states, $\langle n|\mathcal{V}| 0\rangle[47]$. We determined them 
(a)

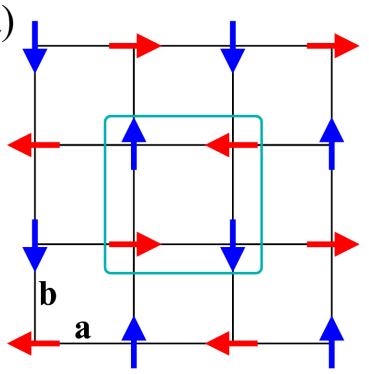

(b)

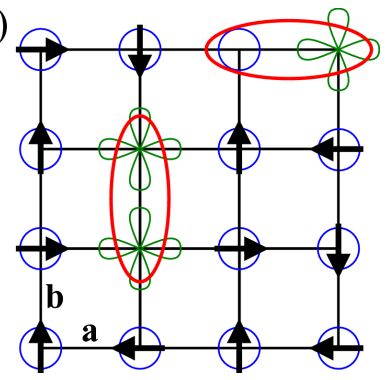

Fig. 5. Artist's view of the exotic spin order found in the ortho-AF phase in the 2D monolayer: (a) one of the two nonequivalent spin configurations, with four spin sublattices - up/down arrows stand for eigenstates of $\left\langle S_{i}^{z}\right\rangle= \pm \frac{1}{2}$, while right/left arrows for $\left\langle S_{i}^{x}\right\rangle= \pm \frac{1}{2}$; (b) orbital $\mathrm{FO} z$ order is locally modified by orbital excitations from $|z\rangle$ (circles) to $|x\rangle$ orbitals (clovers), and the ortho-AF spin order (arrows) is then locally replaced by spin singlets (ovals).

taking certain number of $z$-orbitals flipped to $x$-orbitals and derived their average values. All the averages are taken between orbital states to derive the spin model Eq. (16). Given that $\mathcal{V}$ has non-zero overlap only with states having one or two NN orbitals flipped from $z$ to $x$, one finds in second order effective interactions which couple next nearest neighbors (NNN) and third nearest neighbors $(3 \mathrm{NN})$ in the lattice [47].

The NNN interaction in $H_{s}^{(2)}$ are AF and would give two quantum antiferromagnets on interpenetrating sublattices. To explain the spin ortho-AF order shown in Fig. 5a, i.e., NN spins being perpendicular, one has to include the third order $H_{s}^{(3)}$ as well. Qualitatively new terms as compared to the lower orders arise then [47] with connected products of three different Heisenberg bonds. They provide four-spin couplings and modify the ground state energy. The final result of such an analysis is that the classical energy is indeed minimized by the configurations with angles $\varphi=\pi / 2$ between the NN spins, as shown in Fig. 5a.

It is a challenge to write down the ground state of $H_{s}$ (16), $\left|\mathrm{AF}_{\perp}\right\rangle$, using the described perturbative scheme. It turns out that the quantum corrections obtained within the spin-wave expansion are small and the spin state is nearly classical. Nevertheless, the spins in $H_{s}$ are always dressed with orbital and spin-orbital fluctuations, and the ground state is rather complex. Indeed, within the perturbative treatment one obtains the full spin-orbital ground state shown in Fig. 5b,

$$
\left|\Psi_{\mathrm{SO}}\right\rangle \propto\left(1-\sum_{n \neq 0} \frac{\mathcal{V}_{n}}{\varepsilon_{n}}+\sum_{n, m \neq 0} \frac{\mathcal{V}_{n} \mathcal{V}_{m}}{\varepsilon_{n} \varepsilon_{m}}-\ldots\right)\left|\Phi_{0}\right\rangle,
$$

where $\mathcal{V}_{n} \equiv|n\rangle\langle n| \mathcal{V}, \varepsilon_{n}$ are excitation energies, and $\left|\Phi_{0}\right\rangle \equiv\left|\mathrm{AF}_{\perp}\right\rangle|0\rangle$ is the disentangled classical (Néel-like) state of Fig. 5a. This classical state is dressed with both orbital and spin-orbital fluctuations via the terms which stem from the operator sum in front of $\left|\Phi_{0}\right\rangle$ in Eq. (18). A simpler form is obtained when the purely orbital fluc-

tuations are neglected and density of spin-orbital defects is assumed to be small, one finds

$$
\left|\Psi_{\mathrm{SO}}\right\rangle \simeq \exp \left(-\frac{1}{\left|\varepsilon_{z}\right|} \sum_{\langle i j\rangle|| \gamma} \mathcal{D}_{i j}^{\gamma}\right)\left|\Phi_{0}\right\rangle,
$$

where

$$
\mathcal{D}_{i j}^{\gamma}=\left\{-A \sigma_{i}^{x} \sigma_{j}^{x}+B\left(\sigma_{i}^{x}+\sigma_{j}^{x}\right) s_{\gamma}\right\} \pi_{i j}^{s}
$$

is the spin-orbital excitation operator on the bond $\langle i j\rangle$, with $A=3\left(r_{1}+r_{4}\right) / 2^{6}$ and $B=\sqrt{3}\left(r_{1}+2 r_{2}+3 r_{4}\right) / 2^{5}$. Both terms in Eq. (20) project on a NN spin singlet, but the first one flips two NN $z$-orbitals while the second one generates only one flipped orbital. The density of entangled defects in Fig. 5b increases when $\left|\varepsilon_{z}\right|$ is decreased and the ortho-AF phase is gradually destabilized.

Finally, it is worth to mention that the exotic ortho-AF order was not only predicted by the PMF and explained by the perturbative expansion, but its existence was also corroborated by a variational calculation with the entanglement renormalization Ansatz (ERA). It is remarkable that the range of stability of the ortho-AF phase is similar in both Ansätze in Fig. 6. Actually, this exotic phase is even more robust in the ERA than in the PMF.
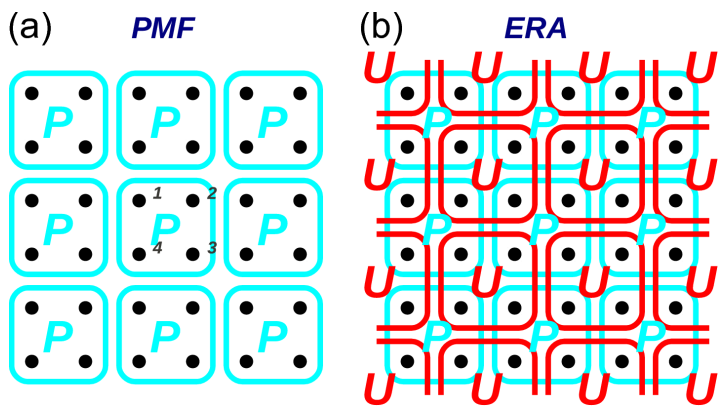

Fig. 6. Two variational Ansätze used to study the 2D ortho-AF phase: (a) the plaquette MF (PMF), and (b) the entanglement renormalization Ansatz (ERA). Black dots are lattice sites, $\mathcal{P}$ 's are variational wave functions on $2 \times 2$ plaquettes, and $\mathcal{U}$ 's are variational $2 \times 2$ unitary disentanglers. The difference between PMF and ERA is the additional layer of disentanglers between the plaquette wave functions $\mathcal{P}$ and the physical degrees of freedom. Their role is to introduce some entanglement between different plaquettes or to disentangle partially the plaquettes before the plaquette product Ansatz is applied. This figure is reproduced from [47].

\subsection{Examples of $3 D$ exotic spin order}

In the $3 \mathrm{D}$ perovskite lattice the ortho-AF phase is found as well in the narrow range of $\left(E_{z}, \eta\right)$ where the spin order changes classically from the $G$-AF to $A$-AF one, and the order is $\mathrm{FO} z$ (Fig. 5a) in $a b$ planes, and $\mathrm{AF}$ between the consecutive planes along the $c$ axis.

When $\eta$ is further increased within the $A$-AF phase, one finds a second magnetic transition, and two exotic phases are found: (i) the striped-AF phase characterized by symmetry breaking between the $a$ and $b$ directions in the orbital and spin sectors for $E_{z}>0$, see Fig. 7a, 
(a)
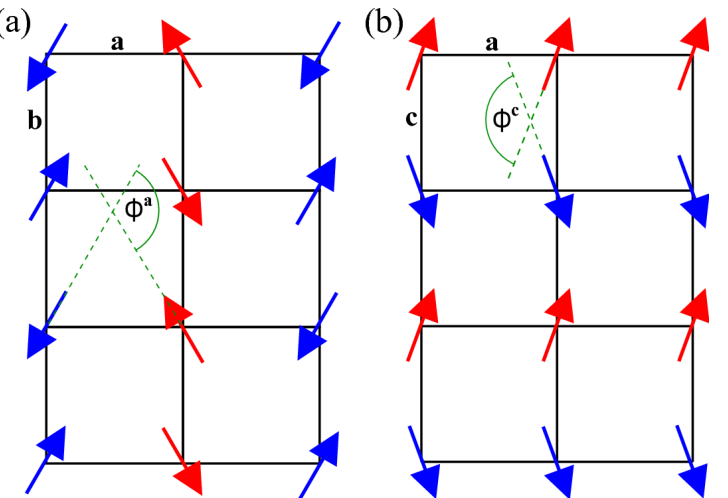

Fig. 7. Two exotic spin orders realized by the $3 \mathrm{D}$ perovskite KK model at large Hund's exchange $\eta>0.2$ : (a) striped-AF order, with AF order along the $c$ axis and angle $\Phi^{a}$ between the NN spins along the $a$ axis (spins are AF along the $b$ axis); (b) canted- $A$-AF phase with FM order in $a b$ planes and spin canting angle $\Phi^{c}$ along the $c$ axis. These phases are precursor states to the FM phase at large $\eta>0.2$, see Fig. 3c.

and (ii) the canted- $A$-AF phase when the spins stay FM within the $a b$ planes, but rotate gradually from the AF to FM configuration along the $c$ bonds with increasing value of $\eta$, see Fig. $7 \mathrm{~b}$. Both phases are characterized by the spin angle - the striped-AF phase by $\Phi^{a}$ along the $a$ axis, and the canted- $A$-AF phase by $\Phi^{c}$ along the $c$ axis. The two phases shown in Fig. 7 are quite different, as the CF term breaks the symmetry in the orbital space.

The transition to the FM order for negative CF splitting involves the intermediate canted- $A-\mathrm{AF}$ phase, shown for $E_{z}=-0.5 J$ in Fig. 8a. Here the spin order is already FM in the $a b$ planes, so it suffices to analyze the phase transition using a linear embedded cluster [49]. The evolution of the spin order is captured by two quantities: the spin canting angle $\Phi^{c}$ along the $c$ axis, and the total magnetization $|s|$, defined as follows:

$$
\begin{aligned}
& \cos \theta=\frac{1}{s^{2}}\left(s_{1}^{x} s_{2}^{x}+s_{1}^{z} s_{2}^{z}\right), \\
& |s| \equiv \sqrt{\left(s^{x}\right)^{2}+\left(s^{z}\right)^{2}} .
\end{aligned}
$$

In the canted- $A$-AF phase $\cos \Phi^{c}$ interpolates smoothly between limits $\cos \Phi^{c}=-1$ in the $A$-AF phase and $\cos \Phi^{c}=1$ in the FM one. Figure 8a shows also that the spin order parameter $|s|$ is almost classical $(|s| \simeq 0.5)$ in the $A$-AF phase as the quantum corrections are here rather low - the quantum spin fluctuations decrease further across the canted- $A$-AF phase and finally one finds the exact value $|s|=0.5$ in the FM phase.

The second example of the exotic spin order found in the 3D KK model considered here is the striped-AF phase which gradually turns into the $G$-AF Néel order when $E_{z}$ increases. In this case the plaquette cluster in an $a b$ plane is more appropriate as it captures the changes of the spin order here. The orbital order parameters $\left\{t^{a}, t^{b}\right\}$ (not shown) suggest the symmetry breaking within the $a b$ planes in the striped-AF phase which is restored at the
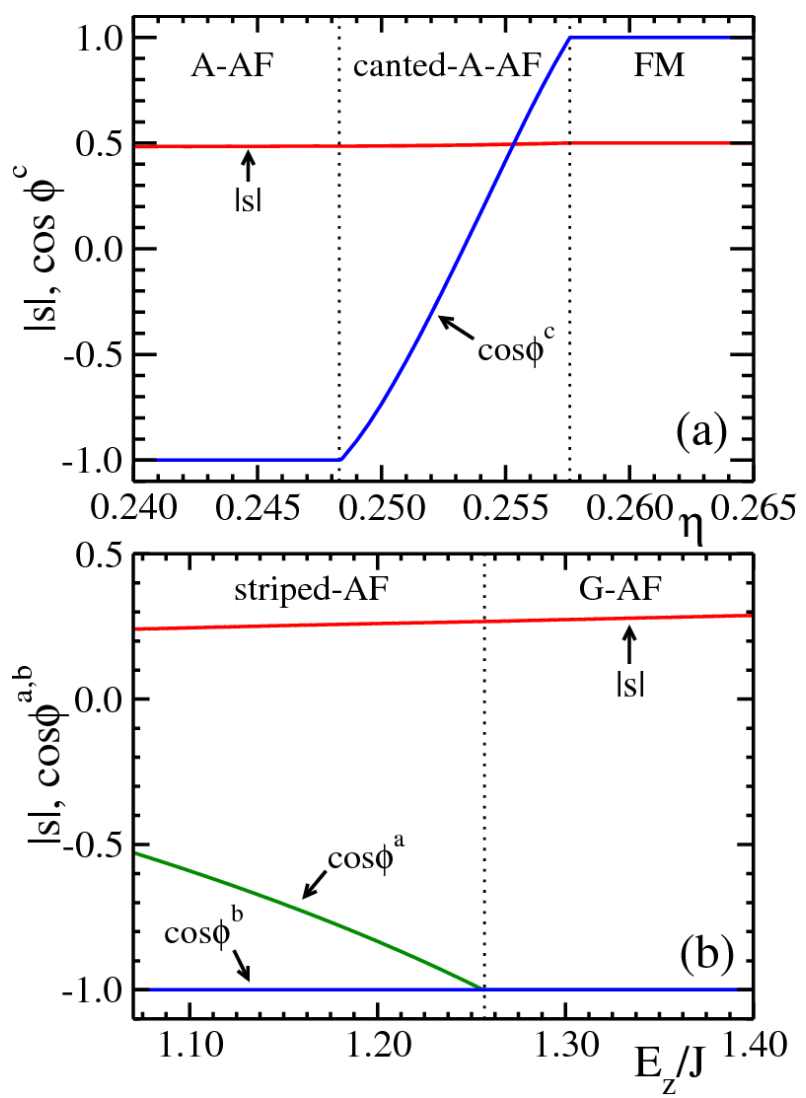

Fig. 8. Spin order parameters $|s|(22)$ and cosines of the angles between two neighboring spins in the two exotic phases found in the 3D perovskite (Fig. 7): (a) evolution from the $A$-AF via the canted- $A$-AF to FM phase with increasing $\eta$ at $E_{z}=-0.5 J$, and (b) evolution from the striped-AF to $G$-AF phase with increasing CF splitting $E_{z} / J$, found at $\eta=0.22$; here spin order $|s|$ is significantly reduced by quantum fluctuations.

transition to the $G$-AF phase. The spin order is given by four sublattices, two of them shown in Fig. 7b, and the other two related to them by spin inversion.

The cosines of two angles between the neighboring spins along the $a$ and $b$ axis $(21), \Phi^{a(b)}$, show that the AF order along the $b$ axis is independent of $E_{z}$, see Fig. 8b. In the $G$-AF phase one finds $\cos \Phi^{a(b)}=-1$, as expected for the uniform antiferromagnet. The total magnetization $0.24<|s|<0.30(22)$ is almost constant and increases monotonically with increasing $E_{z}$. This demonstrates that the essential physics of the striped-AF phase is described by the spin angles, but also that the order parameter is much softened by spin fluctuations on the plaquettes within the $a b$ planes.

\section{Summary}

We have investigated the phase diagrams of the spin-orbital $d^{9}$ Kugel-Khomskii model by the mean-field and perturbative methods for increasing system dimensionality: from the square lattice monolayer, via the bilayer to 
the cubic lattice. In each case we have found strong competition between different types of spin and orbital order, with entangled spin-orbital phases at the crossover from antiferromagnetic to ferromagnetic correlations in the intermediate regime of Hund's exchange. These phases stem from the quantum fluctuations of the ordered orbitals that couple to spins and produce novel types of spin bonds including non-trivial four-spin interactions.

For the 2D monolayer the ortho-AF exotic phase was found, which is characterized by a non-collinear spin order where neighboring spins are perpendicular to each other and orbitals are strongly polarized in a $\mathrm{FO} z$ configuration. Such phases are excluded in the single-site mean-field approach, and could not be found before [51]. Both cluster mean-field and a more involved ERA involving entanglement between clusters confirmed stability of the ortho-AF phase in between the AF and FM phases. On the other hand, the perturbative treatment in the orbital space was introduced to get effective spin Hamiltonian in the ortho-AF phase and to provide the physical insight into mechanism stabilizing spins being at the same time AF on two interpenetrating sublattices and perpendicular for nearest neighbors when virtual orbital flips occur and are accompanied by spin singlets.

The case of a bilayer turned out to be rather special and different from both the $2 \mathrm{D}$ and $3 \mathrm{D}$ case due to its strong tendency towards formation of interplanar singlets. Unlike the ortho-AF phase, the entangled phases found here are located in the intermediate coupling regime and are not triggered by a magnetic phase transition. Apart from this, the location of the longrange order phases and the plaquette valence bond phase is already quite similar to the $3 \mathrm{D}$ case where the orthoAF phase is found again together with two additional phases with exotic spin order induced by orbital fluctuations. These striped-AF and canted- $A$-AF phases appear here at relatively high values of Hund's exchange in the vicinity of the FM phase, and essentially by the same mechanism as the ortho-AF phase in the $2 \mathrm{D}$ case.

The most striking feature in the phase diagram of the $3 \mathrm{D}$ model is that for negative $E_{z}$, the transition from the fully AF to the fully FM configuration takes place gradually with growing Hund's coupling $\eta$, first the $a b$ planes become FM passing through the exotic ortho-AF phase, and next the remaining bonds along the $c$ axis become FM passing through the canted- $A$-AF exotic phase. In contrast, for large positive $E_{z}$ the $\mathrm{AF}$ and FM phases remain unchanged and are connected by a discontinuous transition. The only exception occurs at intermediate $E_{z}>0$ where a striped-AF phase occurs. We argue that this last exotic phase with anisotropic AF order in the $a b$ planes is a positive- $E_{z}$ counterpart of the ortho-AF phase where the effective spin interaction of different orders compete with each other leading to frustration and anisotropy.

Finally, the 2D monolayer system served us as a testing ground of the thermal stability of spin and orbital orders found in the KK model. We have found that orbital order is more robust in general and spin order melts first under increasing temperature, as for instance in $\mathrm{LaMnO}_{3}$ [42]. Robust orbital interactions occur also in triangular lattice, as for instance in $\mathrm{LiNiO}_{2}$ [52]. The most fragile is the exotic spin order found in ortho-AF phase which may be expected taking the energy scales of the effective spin couplings. The other factor that also strongly decreases the temperature of spin melting may be the orbital order which is incompatible with lattice geometry and strongly suppresses in-plane couplings, such as $\mathrm{FO} z$ order found for negative crystal-field $E_{z}<0$. In contrast, the plaquette valence bond phase is a robust type of order due to in-plane singlets. We believe that these general conclusions are valid for other related models as well.

\section{Acknowledgments}

We kindly acknowledge financial support by the Polish National Science Center (NCN) under Projects: No. 2012/04/A/ST3/00331 (W.B. and A.M.O.) and No. 2013/09/B/ST3/01603 (J.D.).

\section{References}

[1] K.I. Kugel, D.I. Khomskii, JETP 37, 725 (1973).

[2] K.I. Kugel, D.I. Khomskii, Sov. Phys. Usp. 25, 231 (1982).

[3] J. van den Brink, Z. Nussinov, A.M. Oleś, in: Introduction to Frustrated Magnetism: Materials, Experiments, Theory, Eds. C. Lacroix, P. Mendels, F. Mila, Springer, New York 2011, p. 631.

[4] G. Khaliullin, Prog. Theor. Phys. Suppl. 160, 155 (2005).

[5] A.M. Oleś, G. Khaliullin, P. Horsch, L.F. Feiner, Phys. Rev. B 72, 214431 (2005).

[6] A.M. Oleś, Acta Phys. Pol. A 115, 36 (2009).

[7] A.M. Oleś, J. Phys. Condens. Matter 24, 313201 (2012).

[8] L.F. Feiner, A.M. Oleś, J. Zaanen, Phys. Rev. Lett. 78, 2799 (1997); J. Phys., Condens. Matter 10, L555 (1998).

[9] G. Khaliullin, V. Oudovenko, Phys. Rev. B 56, R14243 (1997).

[10] Y. Tokura, Prog. Theor. Phys. 69, 797 (2006).

[11] J. Fujioka, T. Yasue, S. Miyasaka, Y. Yamasaki, T. Arima, H. Sagayama, T. Inami, K. Ishii, Y. Tokura, Phys. Rev. B 82, 144425 (2010).

[12] J.-S. Zhou, J.B. Goodenough, Phys. Rev. Lett. 96, 247202 (2006).

[13] K. Wohlfeld, M. Daghofer, S. Nishimoto, G. Khaliullin, J. van den Brink, Phys. Rev. Lett. 107, 147201 (2011).

[14] K. Wohlfeld, S. Nishimoto, M.W. Haverkort, J. van den Brink, Phys. Rev. B 88, 195138 (2013).

[15] A.M. Oleś, P. Horsch, L.F. Feiner, G. Khaliullin, Phys. Rev. Lett. 96, 147205 (2006).

[16] Y. Chen, Z.D. Wang, Q.Y. Li, F.C. Zhang, Phys. Rev. B 75, 195113 (2007). 
[17] B. Normand, A.M. Oleś, Phys. Rev. B 78, 094427 (2008).

[18] J. Chaloupka, A.M. Oleś, Phys. Rev. B 83, 094406 (2011).

[19] A. Herzog, P. Horsch, A.M. Oleś, J. Sirker, Phys. Rev. B 83, 245130 (2011).

[20] W.-L You, A.M. Oleś, P. Horsch, Phys. Rev. B 86 , 094412 (2012).

[21] R. Lundgren, V. Chua, G.A. Fiete, Phys. Rev. B 86, 224422 (2012).

[22] C. Ulrich, G. Khaliullin, J. Sirker, M. Reehuis, M. Ohl, S. Miyasaka, Y. Tokura, B. Keimer, Phys. Rev. Lett. 91, 257202 (2003).

[23] P. Horsch, G. Khaliullin, A.M. Oleś, Phys. Rev. Lett. 91, 257203 (2003).

[24] J. Sirker, A. Herzog, A.M. Oleś, P. Horsch, Phys. Rev. Lett. 101, 157204 (2008).

[25] P. Horsch, A.M. Oleś, L.F. Feiner, G. Khaliullin, Phys. Rev. Lett. 100, 167205 (2008).

[26] B. Normand, Cont. Phys. 50, 533 (2009).

[27] J. van den Brink, P. Horsch, F. Mack, A.M. Oleś, Phys. Rev. B 59, 6795 (1999).

[28] J. van den Brink, New J. Phys. 6, 201 (2004).

[29] A. van Rynbach, S. Todo, S. Trebst, Phys. Rev. Lett. 105, 146402 (2010).

[30] J. Dornier, F. Becca, F. Mila, Phys. Rev. B 72, 024448 (2005).

[31] L. Cincio, J. Dziarmaga, A.M. Oleś, Phys. Rev. B 82, 104416 (2010)

[32] F. Trousselet, A.M. Oleś, P. Horsch, Europhys. Lett. 91, 40005 (2010); Phys. Rev. B 86, 134412 (2012).

[33] W. Brzezicki, A.M. Oleś, Phys. Rev. B 82, 060401 (2010); Phys. Rev. B 87, 214421 (2013).

[34] W. Brzezicki, M. Daghofer, A.M. Oleś, Phys. Rev B 89, 024417 (2014).

[35] B. Frischmuth, F. Mila, M. Troyer, Phys. Rev. Lett. 82, 835 (1999).
[36] A.M. Oleś, P. Horsch, G. Khaliullin, Phys. Status Solidi B 244, 2378 (2007).

[37] B. Kumar, Phys. Rev. B 87, 195105 (2013).

[38] W. Brzezicki, J. Dziarmaga, A.M. Oleś, Phys. Rev. Lett. 112, 117204 (2014)

[39] D.A. Tennant, T.G. Perring, R.A. Cowley, S.E. Nagler, Phys. Rev. Lett. 70, 4003 (1993).

[40] J. Deisenhofer, I. Leonov, M.V. Eremin, Ch. Kant, P. Ghigna, F. Mayr, V.V. Iglamov, V.I. Anisimov, D. van der Marel, Phys. Rev. Lett. 101, 157406 (2008).

[41] B. Lake, D.A. Tennant, J.-S. Caux, T. Barthel, U. Schollwöck, S.E. Nagler, C.D. Frost, Phys. Rev Lett. 111, 137205 (2013)

[42] L.F. Feiner, A.M. Oleś, Phys. Rev. B 59, 3295 (1999).

[43] J.H. Jefferson, H. Eskes, L.F. Feiner, Phys. Rev. B 45, 7959 (1992).

[44] M. Mostovoy, D.I. Khomskii, Phys. Rev. Lett. 89, 227203 (2002).

[45] A.M. Oleś, L.F. Feiner, J. Zaanen, Phys. Rev. B 61 , 6257 (2000).

[46] A.M. Oleś, Phys. Rev. B 28, 327 (1983).

[47] W. Brzezicki, J. Dziarmaga, A.M. Oleś, Phys. Rev. Lett. 109, 237201 (2012).

[48] W. Brzezicki, A.M. Oleś, Phys. Rev. B 83, 214408 (2011).

[49] W. Brzezicki, J. Dziarmaga, A.M. Oleś, Phys. Rev. B 87, 064407 (2013)

[50] K.A. Chao, J. Spałek, A.M. Oleś, J. Phys. C 10, L271 (1977)

[51] J. Chaloupka, G. Khaliullin, Phys. Rev. Lett. 100 016404 (2008).

[52] A.J.W. Reitsma, L.F. Feiner, A.M. Oleś, New J. Phys. 7, 121 (2005). 